\title{
Disorder Limited Exciton Transport in Colloidal Single-Wall Carbon Nanotubes
}

\author{
Jared J. Crochet, ${ }^{* \dagger}$ Juan G. Duque, ${ }^{\dagger}$ James H. Werner, ${ }^{\ddagger}$ Brahim Lounis,,${ }^{\text {II, }}$ \\ Laurent Cognet, ${ }^{\text {II }}{ }^{\S}$ and Stephen K. Doorn*,
}

Physical Chemistry and Applied Spectroscopy, Los Alamos National Laboratory, New Mexico, USA, Center for Integrated Nanotechnologies, Los Alamos National Laboratory, New Mexico, USA, Univ Bordeaux, LP2N, F-3340, Talence, France, and CNRS, Institut d'Optique, LP2N, F-33405, Talence, France

E-mail: jcrochet@lanl.gov; skdoorn@lanl.gov

KEYWORDS: carbon nanotube, exciton, dephasing, transport, exchange interaction

\begin{abstract}
We present measurements of $S_{1}$ exciton transport in $(6,5)$ carbon nanotubes at room temperature in a colloidal environment. Exciton diffusion lengths associated with end quenching paired with photoluminescence lifetimes provide a direct basis for determining a median diffusion constant of approximately $7.5 \mathrm{~cm}^{2} \mathrm{~s}^{-1}$. Our experimental results are compared to model diffusion constants calculated using a realistic exciton dispersion accounting for a logarithmic correction due to the exchange self-energy and a non-equilibrium distribution between bright and dark excitons. The intrinsic diffusion constant associated with acoustic phonon scattering is too large to explain the observed diffusion length, and as such, we attribute the observed

\footnotetext{
${ }^{*}$ To whom correspondence should be addressed

${ }^{\dagger}$ Physical Chemistry and Applied Spectroscopy, Los Alamos National Laboratory, New Mexico, USA

$\ddagger$ Center for Integrated Nanotechnologies, Los Alamos National Laboratory, New Mexico, USA

IUniv Bordeaux, LP2N, F-3340, Talence, France

${ }^{\S}$ CNRS, Institut d'Optique, LP2N, F-33405, Talence, France
} 
transport to disorder-limited diffusional transport associated with the dynamics of the colloidal interface. In this model an effective surface potential limits the exciton mean free path to the same size as that of the exciton wavefunction, defined by the strength of the electron-hole Coulomb interaction.

Single-wall carbon nanotubes are ideal models of infinite $\pi$ orbital conjugation where the rigidity of the $s p^{2}$ lattice gives rise to nearly perfect one-dimensional conducting channels for charge carriers. ${ }^{1}$ Moreover, semiconducting SWNTs provide a unique opportunity for photonics and optoelectronic applications because of well defined optical resonances emanating from onedimensional excitonic states. ${ }^{2}$ The transport of energy in the form of excitons has implications in photovoltaics where both intra- and inter-tube transport are important factors in determining device performance. ${ }^{3}$ There have been several investigations aimed at determining room temperature intratube exciton diffusion lengths $l_{D}$ in SWNTs with the consensus that on average $l_{D}>100 \mathrm{~nm},{ }^{4-10}$ depending on the wrapping surfactant. Indeed, as shown by Siitonen et al ${ }^{7}$ and Gokus $e t a l,{ }^{11}$ both $l_{D}$ and the effective fluorescence lifetimes $\tau_{P L}$ can vary significantly for different environmental conditions. Moreover, the emission line-width of single tubes is also affected by environmental perturbations. ${ }^{12,13}$ Such behavior suggests that optical dephasing, exciton diffusion, and relaxation dynamics are largely tied to the local environment. Most importantly, knowledge of the intricacies of exciton diffusion are crucial for understanding photoexcited energy transport in the context of nanoscale optoelectronics.

With diffusional processes dominating the photophysics of carbon nanotubes, we set out to experimentally determine the exciton diffusion constant in $(6,5)$ SWNTs. To avoid ambiguities associated with fluorescence quenching ranges because of localized and de-localized electronic impurities, ${ }^{10}$ we determined the diffusion lengths directly from exciton quenching associated with the nanotube ends. This ability to directly probe behaviors previously accessed only indirectly is accomplished by imaging long, bright, and stable nanotube emitters at the diffraction limit. Paired with other recent reports, ${ }^{10-13}$ our results highlight the importance of such samples for accessing behaviors typically masked in measurements of shorter and more highly defected tubes. We found 

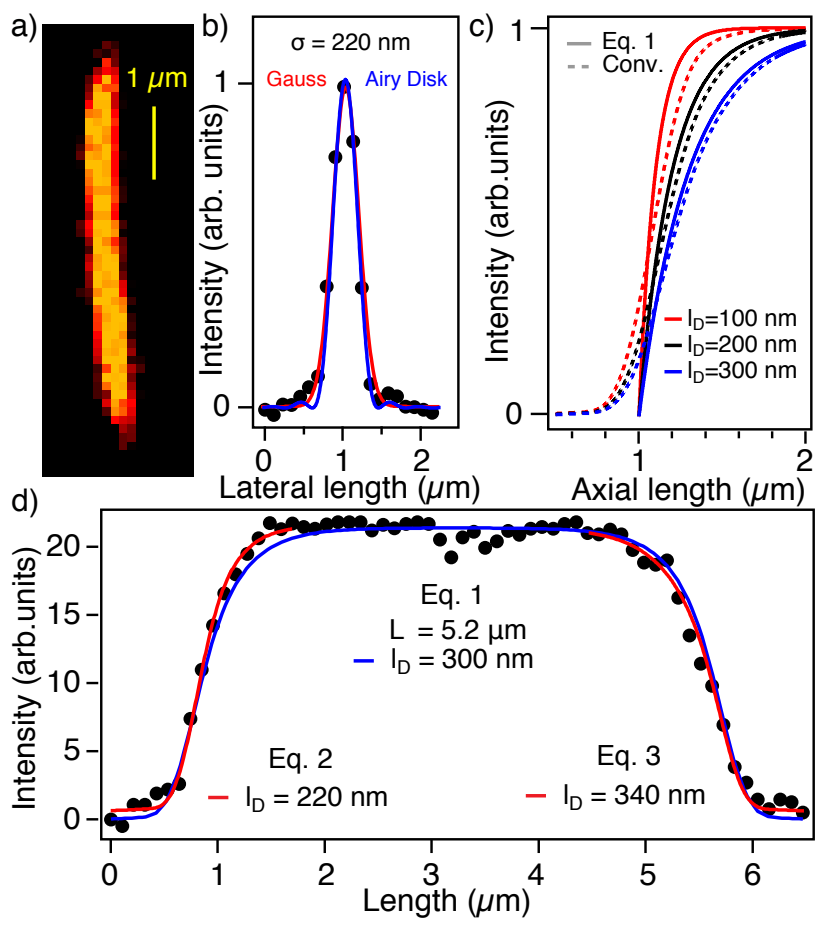

Figure 1: a) Wide field photoluminescence image of an immobilized colloidal $(6,5)$ nanotube. b) Intensity cross-section perpendicular to the tube axis (black circles) from the image in a) with both Gaussian (red) and Airy disk (blue) fits. The Airy disk fit was for $\lambda=986 \mathrm{~nm}$ and $N A=1.49$. This is the imaging point spread function (PSF). c) Simulations of the fluorescence intensity at the end of the tube for various $l_{D}$ (solid lines) and convolved with the PSF (dashed-lines). This illustrates the ability to resolve varying diffusion lengths within our experimental spatial resolution. d) Axial intensity cross-section (black circles) from a) where the fit (red and blue) is the solution of the 1D diffusion equation for different boundary conditions described in the text (Eqs. 1-3) convoluted with the Airy disk in b). 
that for the $(6,5)$ nanotube the median diffusion length was $l_{D}=203 \pm 60 \mathrm{~nm}$, and consistent with previous measurements, ${ }^{11}$ the median fast component of the fluorescence lifetime extracted from a bi-exponential decay was $\tau_{P L}=55 \pm 4$ ps. This corresponds to a median exciton diffusion constant of $D=7.5 \pm 2.3 \mathrm{~cm}^{2} \mathrm{~s}^{-1}$. Our experimentally obtained diffusion constant is examined against diffusion constants calculated within models of non-equilibrium band mediated transport initiated by acoustic phonons versus a mechanism mediated by disordered interface scattering.

Two samples of HiPco synthesized nanotubes (batch \# 187.4) were suspended in $1 \%$ aqueous deoxycholate (DOC) by shear mixing. Un-solubilized material was removed from the suspension by bench-top centrifugation. Imaging was performed on one sample with an inverted microscope equipped with an electron-multiplying CCD camera (Princeton Instruments ProEm) and a 1.49 NA 60x objective in a wide field geometry, where the total detection efficiency was estimated to be $\approx 1.3 \%$. The excitation source consisted of a continuous-wave solid-state $561 \mathrm{~nm}$ laser diode where the photon flux density was kept at $7 \times 10^{20} \mathrm{~cm}^{-2} \mathrm{~s}^{-1}$. This excitation is slightly off resonant from the $S_{2}$ maximum of the $(6,5)$ tube and we can expect a reduction of the absorption cross section per atom by $\approx 30 \%$ from $1 \times 10^{-17} \mathrm{~cm}^{2}$ to $7 \times 10^{-18} \mathrm{~cm}^{2}{ }^{14}$ Spectroscopy and lifetime measurements were carried out on the other sample of SWNTs that was immobilized in aqueous agarose gels (5 wt \%). The dispersion was sandwiched between a glass coverslip and a glass slide and excited confocally at $567 \mathrm{~nm}$ with a dye laser which is resonant with $S_{2}$ of the $(6,5)$ tube. The luminescence was sent to a cryogenically cooled 1D InGaAs detector (OMA V, Roper Scientific) placed at the output of a spectrometer. Lifetime measurements were carried out with by confocal excitation at $567 \mathrm{~nm}$ using a femtosecond optical parametric oscillator and the luminescence decays were recorded using conventional time-resolved single-photon counting.

Fig. 1a shows a photoluminescence image of an approximately $6 \mu \mathrm{m}$ SWNT. The PL image was uniform over the length of the tube and did not show any signs of blinking or quenching except at the tube ends. The quantum yield of this particular tube was estimated to be a few percent using the corrected photon count rate from the camera, the exposure time, and the corrected absorption cross section stated earlier. The point spread function PSF of our microscope was determined by 
fitting the intensity profile perpendicular to the tube axis in Fig. 1a with either a Gaussian of width $\sigma=220 \mathrm{~nm}$ or a slice of an Airy disk which describes the diffraction limit. Using the numerical aperture of the objective $N A=1.49$ and the wavelength of the emitted light $\lambda=986 \mathrm{~nm}$ we found a nearly perfect fit to the lateral intensity trace in Fig. 1b with the Airy disk, indicating a diffraction limited image.

From this point forward we discuss the diffusive behavior of excitons in nanotubes where their concentration is low, given by our excitation rate of a few excitons per ns. In this regime excitonexciton interactions are negligible. If the excitons are truly behaving as one-dimensional diffusers such that the tube ends completely quench the fluorescence and the lifetime distribution of the excitons can be approximated by a single exponential $e^{-t / \tau_{P L}}$, the axial intensity is then given by the solution to the time-independent one-dimensional diffusion equation $D c^{\prime \prime}=-c / \tau_{P L}+S$. Here, $c$ is the exciton concentration, $S$ is the excitation rate, $D$ is the exciton diffusion constant, $\tau_{P L}$ is the effective exciton lifetime, and the double prime is the Laplacian. With the boundary conditions $c(x \leq 0)=c(\geq L)=0$ and the emission intensity is uniform across the tube length as well as proportional to the steady-state exciton concentration, given by $S \tau_{P L}$, the solution takes the form,

$$
c(x)=\left[1-\frac{\sinh \left(x / l_{D}\right)+\sinh \left[(L-x) / l_{D}\right]}{\sinh \left(L / l_{D}\right)}\right],
$$

where $L$ is the length of the tube, $l_{D}=\sqrt{D \tau_{P L}}$ is the exciton diffusion length, and $S \tau_{P L}$ is set to unity which normalizes the equation. We tested the feasibility of using Eq. 1 convoluted with the $P S F$ to discern different diffusion lengths by simulating the expected response for various $l_{D}$ 's, Fig. 1c. We found we could clearly make a distinction between $l_{D}$ varying by $\pm 50 \mathrm{~nm}$. As an example of the fitting process applied to our experimental data for a tube with uniform brightness, we obtain a good fit to the axial trace of the tube shown in Fig. 1a with $l_{D}=300 \mathrm{~nm}$ and $L=5.2 \mu \mathrm{m}$, Fig. 1d. However, the realistic situation is where tubes are not uniformly emitting and each end has a slightly different environment which would result in different exciton transport properties. In this case the boundary condition for a half space must be used i.e. $c(\leq 0)=0$ and $c(x>0)=S \tau_{P L}$ for the left end and $c(x>L)=S \tau_{P L}$ and $c(x \geq L)=0$ for the right end of the tube. The normalized 
$\left(S \tau_{P L}=1\right)$ solution to the diffusion equation then becomes,

$$
c(x)=\left[1-\frac{\sinh \left[(L-x) / l_{D}\right]}{\sinh \left(L / l_{D}\right)}\right]
$$

for the left end of the tube and,

$$
c(x)=\left[1-\frac{\sinh \left(x / l_{D}\right)}{\sinh \left(L / l_{D}\right)}\right]
$$

for the right end of the tube. In Fig. 1d we show the comparison of using Eqs. 1-3 and we obtain $l_{D}=220 \mathrm{~nm}$ for the left end and $l_{D}=340 \mathrm{~nm}$ for the right end of the tube. A better fit to the intensity decays is obtained if each end of the tube is fit independently. This is evidenced by the fact a distribution of diffusion lengths is needed to fit all the tubes and each tube end (Fig. 2b). As a complement to the simulated intensity data in Fig. 1c, we demonstrate for our experimental data the ability to discriminate between differing $l_{D}$ 's in Fig. 2 a.

We examined end quenching from 55 tubes by using Eqs. 2 and 3 to find a median $l_{D}$ that can represent an ensemble of tubes.The diffusion length was found to vary from tube-to-tube, and a histogram of all determined $l_{D}$ 's is shown in Fig. $2 \mathrm{~b}$ and was found to have a median of $l_{D} \approx$ $203 \pm 60 \mathrm{~nm}$. Here the histogram bin width was chosen such that the integrated mean squared error was minimized. ${ }^{15}$ This result is consistent with single molecule step-wise quenching experiments for the same encapsulating surfactant, ${ }^{7}$ which not only provides validity to extracting $l_{D}$ from step-wise quenching, but also suggests that $l_{D}$ may be independent of the quenching mechanism for localized electronic impurities such as mid-gap states or tube ends. ${ }^{10}$ Moreover, our result is nearly the same as the diffusion length of excitons in larger diameter tubes in laboratory air, ${ }^{16}$ which suggests excitons may experience similar transport mechanism in atmospheric environments where several monolayers of adsorbates are present.

In Fig. 3a we show a fluorescence lifetime for a single $(6,5)$ tube with it's associated fit. The time dependence of the fluorescence intensity is systematically bi-exponential and fit by $I(t)=$ $\left(A e^{-t / \tau_{1}}+B e^{-t / \tau_{2}}\right)$ convoluted with the instrument response function (IRF) of the time-correlated 
single photon counting system. ${ }^{11,14}$ As reported previously, ${ }^{11,14}$ the long component $\tau_{2}$ with a weight of $B \tau_{2} /\left(A \tau_{1}+B \tau_{2}\right)$ accounts for $\approx 10 \%$ of the total fluorescence intensity. The effective fluorescence lifetime was then calculated as a weighted average $\tau_{P L}=\int I(t) d t=\left(A \tau_{1}+B \tau_{2}\right) /(A+B)$, and represents the effective bright exciton lifetime. ${ }^{14}$ In Fig. $3 \mathrm{~b}$ a histogram of $\tau_{P L}$ 's collected from $17(6,5)$ tubes is shown. A median $\tau_{P L}$ of $55 \pm 4$ ps was deduced, and the diffusion constant, $D=l_{D}^{2} / \tau_{P L}$, was determined to be $D=7.5 \pm 2.3 \mathrm{~cm}^{2} \mathrm{~s}^{-1}$. This is in fair agreement with indirect measurements using a diffusion limited contact quenching model applied to fluorescence lifetimes and intensities of length sorted $(6,5)$ tubes. ${ }^{8}$ Moreover, when compared to other indirect determinations of the diffusion constant ${ }^{5,7,16}$ we find good agreements with our result if an accurately measured $\tau_{P L}$, appropriate to the specific system under study, is applied. For example, the original result of Ref. 5 ( $\left.D=0.4 \mathrm{~cm}^{2} \mathrm{~s}^{-1}\right)$ concerned sodium dodecyl benzene sulfonate (SDBS) dispersed nanotubes and relied on the use of a $\tau_{P L}$ of $100 \mathrm{ps,} \mathrm{originally} \mathrm{estimated} \mathrm{as} \mathrm{a} \mathrm{median} \mathrm{value} \mathrm{from}$ several literature results. ${ }^{5}$ If instead a more current value for $\tau_{P L}$ of $53 \mathrm{ps}$ in $\mathrm{SDBS}^{14}$ is used, and by accounting for the effect of different surfactant environments (SDBS vs. our use of DOC), ${ }^{7}$ a comparable $D$ of $5.4 \mathrm{~cm}^{2} \mathrm{~s}^{-1}$ results. Similarly, indirect determinations of $D=2.8 \mathrm{~cm}^{2} \mathrm{~s}^{-1}$ (in DOC) $)^{7}$ and of $4.7 \mathrm{~cm}^{2} \mathrm{~s}^{-1}$ (for individual tubes suspended in air) ${ }^{16}$ result from $\tau_{P L}$ of $50 \mathrm{ps}^{11,12}$ and $85 \mathrm{ps},{ }^{17}$ respectively, obtained from similar sample types. These indirect determinations are thus seen to be in good agreement with our direct evaluation of $D$.

With this directly obtained diffusion constant we now investigate the fluorescence linewidth, which contains information about the exciton dephasing mechanism. Exciton dephasing, which drives transport by changing the momentum and consequently the group velocity of the exciton, should vary significantly for different environments. Indeed, recent time-resolved coherent spectroscopies of $(6,5)$ nanotubes in various matrices have shown that this is the case. ${ }^{18}$

The fluorescence linewidth at the single tube level, given by $\Gamma=\hbar\left[\tau_{P L}^{-1}+2 \tau_{\phi}^{-1}\right]$, where $\tau_{P L}$ is the effective lifetime or population decay and $\tau_{\phi}$ is the pure dephasing time describing the mean free time between decohering scattering events, was recently found to vary linearly over a wide range of temperatures in vacuum. ${ }^{19}$ This was attributed to a small amount of constant environ- 

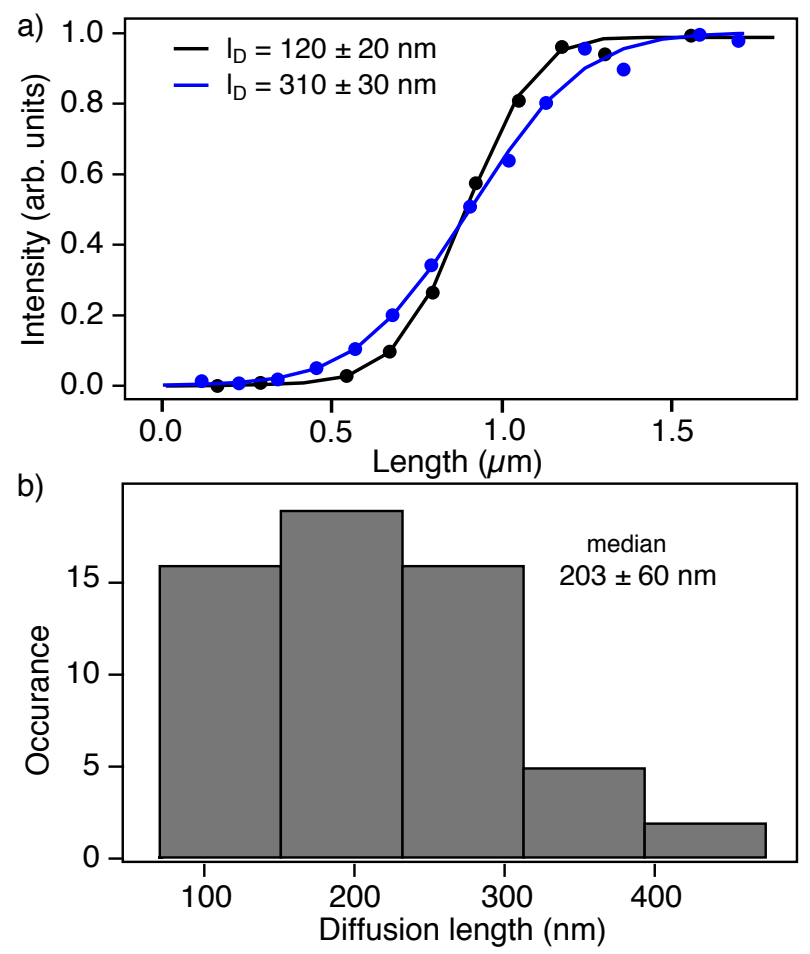

Figure 2: a) Axial intensity traces from the ends of two different (6,5) SWNTs and fits using Eq. 2 for $l_{D}$. The data were horizontally scaled such that an intensity of 0.5 was at the same x-position. b) Histogram of diffusion lengths for 59 tubes. A median diffusion length of $l_{D} \approx 203 \pm 60 \mathrm{~nm}$ was determined. 
mental dephasing of $\Gamma_{0} \approx 2 \mathrm{meV}$ and an exciton-acoustic phonon coupling term that lead to a diameter dependent $\Gamma$. In comparison, in Fig. 3c we show an emission spectrum for a single $(6,5)$ tube with its associated Voigt fit as described by Cambre et al, ${ }^{13}$ and find that the Gaussian component, inhomogeneous broadening, has a width of $\gamma_{G} \approx 14 \mathrm{meV}$ and the Lorentzian component, homogeneous broadening, has a width of $\gamma_{L} \approx 13 \mathrm{meV}$. We note that a pure Lorentzian line shape gives an approximate $20 \%$ increase in the root-mean-square error associated with the fit. This is consistent with the values obtained from a similar synthesis batch of $(6,4)$ tubes, ${ }^{13}$ and suggests that inhomogenous broadening due to environmental dephasing may be significant in the colloidal environment. In Fig. 3b a histogram of pure exciton dephasing times calculated from several Lorentzian components of the emission linewidths is shown: $\tau_{\phi}=2 \hbar / \gamma_{L}$. The median was found to be $\tau_{\phi}=115 \mathrm{fs}$, and is very similar to that found for the same diameter of tubes suspended over trenches, ${ }^{19}$ where inhomogenous broadening is expected to be less significant. Interestingly, it was found that for colloidal tubes from the same synthesis batch, the emission intensity varied significantly for tubes suspended in two different surfactant systems, while $\Gamma$ was nearly unchanged. ${ }^{12}$ Only for highly defective tubes was $\Gamma$ shown to change significantly and attributed to additional lattice defect dephasing. However, the large spread in the data and the significant increase in inhomogeneous broadening on going from vacuum suggests local environmental fluctuations at room temperature introduce a significant degree of disorder as expected from the dynamic nature of the colloidal interface. Pairing the pure dephasing time with our experimentally determined diffusion constant allows us to address the possible mechanisms behind exciton diffusion in colloidal nanotubes. We proceed to quantify the transport mechanism in $(6,5)$ nanotubes using what is known about the band structure and bright and dark exciton scattering.

In chiral carbon nanotubes there are two distinct $A$ line group symmetry excitons split by an exchange energy of $\Delta \approx 5 \mathrm{meV}$ that have even $A_{2}$ and odd parity $A_{1} \cdot{ }^{20,21}$ The higher energy $A_{1}$ exciton carries all of the oscillator strength and therefore is optically bright, while the lower energy $A_{2}$ exciton is dark. It is also known that the bright exciton dispersion $\varepsilon_{b, q}$, which dictates transport properties through the group velocity, is non-parabolic because of the exchange interaction and 
varies as $\varepsilon_{b, q}=\alpha q^{2} \log \left(q_{0} /|q|\right)$, where $q_{0}$ is a momentum cutoff, $q$ is the center of mass momentum, and $\alpha$ is a coupling constant that measures the strength of the exchange energy. ${ }^{20,22}$ Here $\varepsilon_{b, q}$ is singular at $q=0$, however the singularity is removable as $\lim _{q \rightarrow 0} \varepsilon_{b, q}=0$. Therefore, the diffusion constant of excitons in SWNTs should be treated carefully since the effective mass has a singular logarithmic correction as $q \rightarrow 0$. Recently this behavior has been explained in terms of the spin-charge velocity separation aspect of the Luttinger liquid, ${ }^{23}$ and has roots in the quasi-particle renormalization of the graphene dispersion which does not exactly cancel in one-dimension. ${ }^{24}$ We find the parameters $\alpha$ and $q_{0}$ by fitting $\varepsilon_{b, q}$ to the thermally accessible momentum states by the predictions of Jiang et al for the $(6,5)$ tube calculated using the Bethe-Salpeter equation: ${ }^{20}$ $\alpha=2.85 \mathrm{eV}-\mathrm{nm}^{2}$ and $q_{0}=1.4 \mathrm{~nm}^{-1}$. The dark exciton, however, has a dispersion of a free particle, $\varepsilon_{d, q}=\hbar^{2} q^{2} / 2 m^{*}$, with an effective mass given by $m^{*} \approx 1.5\left(m_{e}^{*}+m_{h}^{*}\right)$ where $m_{e}^{*}$ and $m_{h}^{*}$ are the effective masses of the electron and hole. ${ }^{22}$ From this point forward, we take for the $(6,5)$ tube $m_{e}^{*}+m_{h}^{*}=0.29 m_{e} \cdot{ }^{25}$ Both $\varepsilon_{d}$ and $\varepsilon_{b}$ are shown in Fig. 4a where the bright state is scaled by the optical gap $\varepsilon_{g}$ and the dark exciton scaled by $\varepsilon_{g}-\Delta$.

It is also known that the $A_{2}$ and $A_{1}$ symmetry excitons can inter-parity scatter due to $A$ line group symmetry breaking with rates given by $\gamma_{\uparrow}$ and $\gamma_{\downarrow}$, however, this process is known to be slow when compared to the lifetimes of both parity excitons. ${ }^{11,14}$ Here $\gamma_{\uparrow}=\gamma_{0} n(\Delta)$ and $\gamma_{\downarrow}=\gamma_{0}[n(\Delta)+$ 1], where $n$ is the Bose-Einstein distribution function and $\gamma_{0}$ is the zero temperature scattering rate. For small $\gamma_{0}$ compared to other decay processes this behavior results in a non-equilibrium distribution between the states largely responsible for the bi-exponential behavior shown in Fig. $3 \mathrm{a},{ }^{11,13}$ and has also been shown directly by low-temperature photoluminescence spectroscopy at the single tube ${ }^{26}$ and ensemble levels. ${ }^{27}$ To account for the distribution between $A_{1}$ and $A_{2}$ excitons as well as other processes including: the generation $S_{b}$ and effective decay $\gamma_{b}$ of $A_{1}$ excitons, and the generation $S_{d}$ and effective decay $\gamma_{d}$ of $A_{2}$ excitons; the population of the bright state at $q=0$ is calculated by the steady state solution to the system in Fig 4a such that,

$$
n_{b}=\frac{\gamma_{\uparrow} S_{d}+\left(\gamma_{d}+\gamma_{\uparrow}\right) S_{b}}{\gamma_{d} \gamma_{\downarrow}+\gamma_{d} \gamma_{b}+\gamma_{\uparrow} \gamma_{b}} .
$$



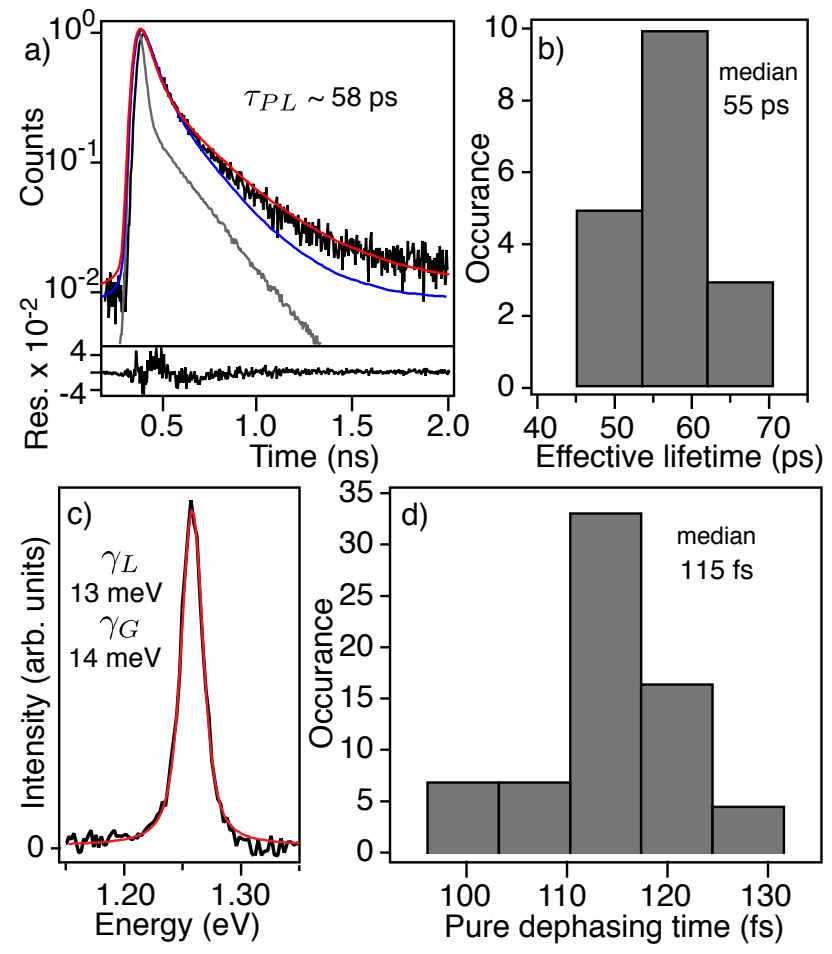

Figure 3: a) Photoluminescence lifetime from a single (6,5) tube (black), instrument response function (grey), and double exponential (red) and mono-exponential fits (blue). The double exponential fit is used to determine the effective fluorescence lifetime, $\tau_{P L}$, where $\tau_{1} \approx 49 \mathrm{ps}$ and $\tau_{2} \approx 449 \mathrm{ps}$. The residual of the double exponential fit is shown at the bottom of $3 \mathrm{a}$ and we consistently find that $\approx 90 \%$ of the intensity is comprised of the fast component. The mono-exponential fit has a time constant of $\tau \approx 58 \mathrm{ps}$. b) Histogram of effective lifetimes $\tau_{P L}$ where the median is $\tau_{1}=55 \pm 4$ ps. For details see Gokus et. al. ${ }^{11}$ c) Fluorescence spectrum from a single $(6,5)$ tube (black) and a Voigt fit (red) used to determine the pure dephasing time, $\tau_{\phi}$, from the Lorentzian component $\gamma_{L}$. The inhomogenous broadening is given by the Gaussian component $\gamma_{G}$. The errors in the linewidths are less the $1 \%$. d) Histogram of pure dephasing times calculated from the linewidth $\gamma_{L}=2 \hbar \tau_{\phi}^{-1}$. The median is $\tau_{d}=115 \mathrm{fs}$. 
Likewise, the lower energy $A_{2}$ exciton population becomes,

$$
n_{d}=\frac{\gamma_{\downarrow} S_{b}+\left(\gamma_{b}+\gamma_{\downarrow}\right) S_{d}}{\gamma_{d} \gamma_{\downarrow}+\gamma_{d} \gamma_{b}+\gamma_{\uparrow, q} \gamma_{b}} .
$$

This allows us to define the total exciton fraction in the bright and dark states as $\eta_{b}=n_{b} /\left(n_{b}+n_{d}\right)$ and $\eta_{d}=n_{d} /\left(n_{b}+n_{d}\right)$. We calculated the populations in each state by assuming the exciton generation rates take the form $S_{b}=S_{d}{ }^{11}$ This is valid under low intensity or continuous wave excitation, the initial population of both states is equal upon internal conversion from higher lying excited states such as $S_{2}$, and the exciton dephasing mechanism is much faster than any of the scattering mechanisms in Fig. 4a, leading to a rapid intra-band thermalization. Using parameters that describe the bi-exponential decay of the photoluminescence: $\gamma_{d}=2 \mathrm{~ns}^{-1}, \gamma_{b}=20 \mathrm{~ns}^{-1}$, and $\gamma_{0}=0.05 \mathrm{~ns}^{-1},{ }^{11}$ we find at steady state the probability of finding an exciton in the bright state $\eta_{b} \approx$ $11 \%$ is much smaller than the probability of finding an exciton within the dark state $\eta_{d} \approx 89 \%$. Moreover, if we take $\gamma_{0}=0$ the probabilities become $\eta_{b} \approx 9 \%$ and $\eta_{d} \approx 91 \%$, which illustrates that the weak, but finite inter-band scattering results in an $\approx 2 \%$ increase of the population of excitons in the bright state. Therefore, at room temperature inter-parity scattering has an insignificant effect on thermally averaged observables since at steady state $\approx 98 \%$ of the bright excitons originated in the bright state and only $\approx 2 \%$ come from the dark state. Within this argument, including only rapid intra-band thermalization, we can define $q$ dependent probability distribution functions of the excitons as $N_{i, q}=e^{-\beta \varepsilon_{i, q}} / \sum_{q} e^{-\beta \varepsilon_{i, q}}$, where $i=b$ or $d$ and $\beta=k_{b} T$. In Fig. 4b we show $N_{b, q}$ and $N_{d, q}$ calculated at room temperature. As a first implication of this model, from $N_{b, q}$ and the effective mass $m^{*}=\hbar^{2}\left|\frac{\partial^{2} \varepsilon_{b}}{\partial q^{2}}\right|^{-1}$, we calculated the expected thermally averaged mass of bright excitons as $\left\langle m^{*}\right\rangle$, where $\langle\ldots\rangle=\int_{-\infty}^{\infty} N_{b, q} \ldots d q$, and find at room temperature $\left\langle m^{*}\right\rangle \approx 0.02 m_{e}$. This is an essential point because $m *$ itself is $q$ dependent and $m^{*} \rightarrow 0$ as $q \rightarrow 0$, Fig. 4c. Also the exciton mass at $\varepsilon_{b, q}\left(q_{c}\right)$, where $q_{c}$ is the wavevector where the dispersion of light intersects the exciton band, is significantly smaller than $\left\langle m^{*}\right\rangle$.

Next we investigated the mechanism of exciton transport by considering two different processes for exciton diffusion: transport by scattering with intrinsic phonons and disorder limited transport 

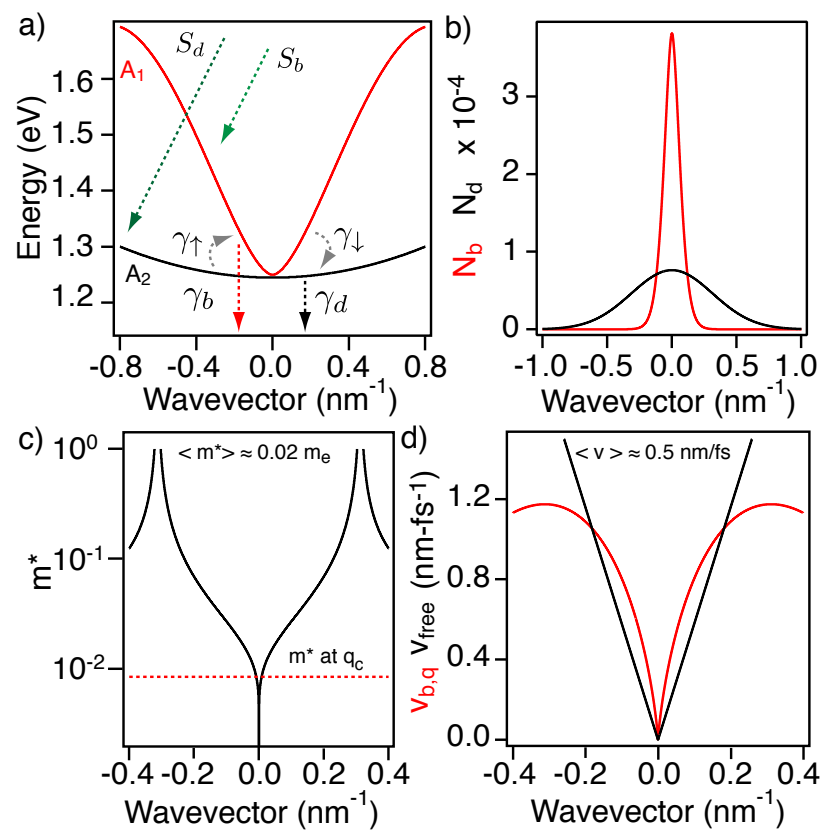

Figure 4: a) Exciton dispersions used in the calculation of the diffusion constant. The bright $A_{1}$ symmetry exciton is shown in red and the dark $A_{2}$ symmetry exciton is shown in black. The rates used to calculate the bright exciton distribution are also shown, where $\gamma_{\downarrow}$ and $\gamma_{\uparrow}$ are the $A_{1} / A_{2}$ inter-parity scattering, $\gamma_{b}$ is the $A_{1}$ exciton decay dominated by non-radiative processes, $\gamma_{d}$ is the $A_{2}$ exciton decay, and $S_{b} / S_{d}$ represents $A_{1} / A_{2}$ exciton generation rates. b) $q$ dependent probability distribution functions calculated from intra-band thermalization only. c) $q$ dependent bright exciton effective mass. The red dashed line illustrates the mass where the dispersion of light intersects the exciton band. d) $q$ dependent exciton velocities calculated with the exchange correction to the dispersion (red) and an effective mass exciton $m^{*}=0.02 m_{e}$ with a parabolic dispersion (black). 
by interfacial scattering. In the first case, the transport is governed by the scattering of excitons within the band $\varepsilon_{b, q}$ by low energy phonons such as acoustic phonons, Fig. 5a. Here the diffusion constant can be calculated from the exciton group velocity,

$$
v_{b, q}=\hbar^{-1} \frac{\partial \varepsilon_{b, q}}{\partial q}
$$

such that $D=\left\langle v_{b, q}^{2} \tau_{\phi}\right\rangle \cdot{ }^{28}$ In Fig. 4d we show $\left|v_{b, q}\right|$ for the logarithmically corrected dispersion compared to the velocity of a free particle $\left|v_{q, \text { free }}\right|=\hbar|q| / m^{*}$ with $m^{*}=0.02 m_{e}$, and it is apparent how the velocity at small $q$ is significantly increased because of the exchange self-energy. Also, we note that in the limit of a single parabolic exciton band of constant mass $m^{*}$ with $q$ independent dephasing and a Boltzmann probability distribution function, the diffusion constant in this description gives the familiar result for a classical particle, $D=k_{b} T \tau_{\phi} / m^{*}$, which is often used for describing excitons in carbon nanotubes. However, we find that for the measured median exciton dephasing time and the logarithmically corrected dispersion $D \approx 370 \mathrm{~cm}^{2} \mathrm{~s}^{-1}$. With this diffusion constant the corresponding mean free path, average distance traveled before a change in momentum or coherence length, ${ }^{29}$ due to acoustic phonon scattering would be $\xi=\sqrt{2 D \tau_{\phi}} \approx 90 \mathrm{~nm}$ and the diffusion length would be $l_{D} \approx 1400 \mathrm{~nm}$. This diffusion length is larger than what we obtain experimentally and suggests this type of transport does not dominate in the colloidal environment.

Next, we examined the effects of interface scattering or a disordered environment on the diffusion constant. In this regime, an exciton wavepacket moves with a certain transport velocity $v$ and is scattered by an inhomogeneous surface potential provided by the dynamic colloidal interface, Fig. 5b. Such motion is derived from standard theory of waves propagating in strongly scattering media and is described by a mean free path $\xi$ such that $D=v \xi .{ }^{30}$ Here, the transport velocity is given by the thermally averaged group velocity $v=\left\langle\left|v_{b, q}\right|\right\rangle$ and at room temperature we find for the logarithmically corrected dispersion $v \approx 0.5 \mathrm{~nm}-\mathrm{fs}{ }^{-1}$. In order for $D \approx 7.5 \mathrm{~cm}^{2} \mathrm{~s}^{-1}$ the mean free path must be $\xi \approx 1.5 \mathrm{~nm}$. As a theoretical comparison, the predicted exciton correlation length, full width-half maximum of the nearly Gaussian envelope function, of a $(6,5)$ nanotube embedded in an effective dielectric constant of $\kappa=1.85$ is $\sigma \approx 1.5 \mathrm{~nm} .{ }^{31}$ Moreover, an experimentally de- 


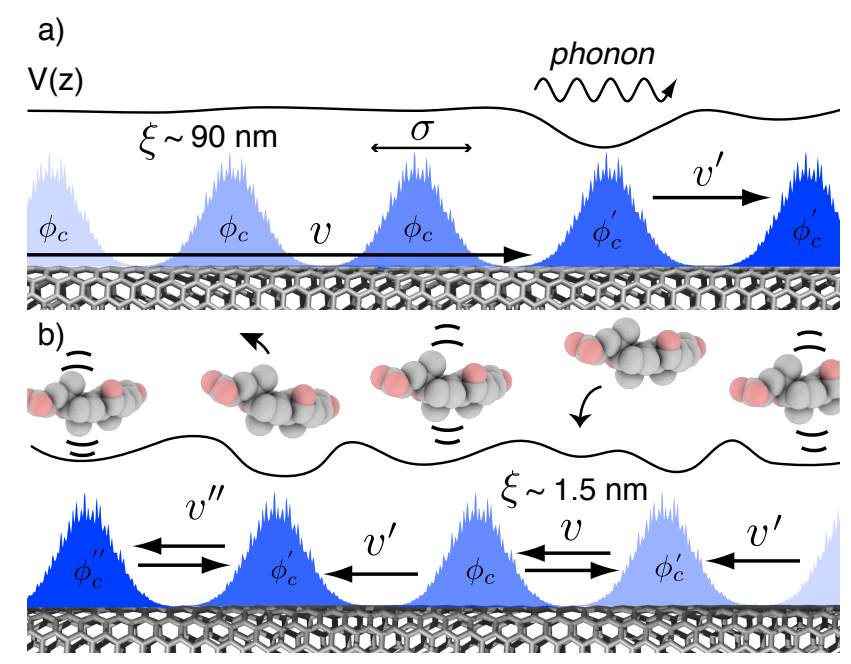

Figure 5: Schematic of exciton transport mechanisms in carbon nanotubes. a) Intrinsic case where an exciton wave-packet, descried by a Gaussian electron-hole pair probability distribution of width $\sigma$, moves with a velocity $v$ and scatters at a lattice site through the deformation potential where phonon absorption or emission takes place and changes the exciton velocity to $v^{\prime}$. Within the coherence length $\xi$ the phase of the wavefunction $\phi_{c}$ is preserved. b) The disorder limited case where a dynamic, random surface potential $V(z)$ associated with the colloidal interface facilitates exciton scattering between energy minima spaced by an average distance that defines the coherence length $\xi$.

termined exciton wave-function with a Gaussian full-width half maximum of $\sigma=2 \pm 0.7 \mathrm{~nm}$ has been measured by phase-space filling. ${ }^{32}$ This suggests that the exciton travels, on average, a distance equal to the width of a distribution function describing the probability to find an electron or hole at a fixed hole or electron position. Moreover, excitons are coupled to the local environment such that a scattering event can take place on average every distance $\xi$ which gives an upper estimate to the linear packing density of the colloidal interface. In this description a larger diffusion constant would suggest a less disordered interface and may be experimentally tested by varying the surfactant composition and concentration.

In conclusion, we have measured the exciton diffusion constant in colloidal $(6,5)$ carbon nanotubes by a combination of photoluminescence imaging and time-resolved fluorescence microscopies. The mean diffusion constant was found to be $D \approx 7.5 \mathrm{~cm}^{2} \mathrm{~s}^{-1}$ and attributed to disorder limited exciton transport. The mean free path $\xi$ we obtained suggests that the exciton moves a distance comparable to it's electron-hole correlation length $\sigma$ before scattering to a new velocity 
and supports an apparent diffusive "hopping" behavior of excitons in colloidal nanotubes. Moreover, our model of exciton transport accounts for what is known theoretically about excitons in carbon nanotubes beyond the simple classical particle picture, which ignores the exchange selfenergy. ${ }^{24}$ This work is also important for understanding the impact of the colloidal interface for the photophysics of carbon nanotubes for single molecule detection applications. ${ }^{10}$ For example, we point out how the mobility of photoexcitations can be altered by effectively changing the surface potential experienced by the excitons and may allow for intrinsic transport. Such insight is of value for colloidal science where the excitons act as a surface probe and we expect by tailoring the composition and structure of the interface, tunability of the optical properties of carbon nanotubes can be realized.

\section{Acknowledgement}

J.C. thanks Jay D. Sau for stimulating theoretical discussions. This work was performed, in part, at the Center for Integrated Nanotechnologies, a U.S. Department of Energy, Office of Basic Energy Sciences user facility and partially supported by LANL-LDRD program. Los Alamos National Laboratory is operated by Los Alamos National Security, LLC, for the National Nuclear Security Administration of the U.S. Department of Energy under contract DE-AC52-06NA25396. This work was also funded by the Agence Nationale de la Recherche, Région Aquitaine, the French Ministry of Education and Research, and the European Research Council.

\section{References}

(1) Avouris, P.; Appenzeller, J.; Martel, R.; Wind, S. P. IEEE 2003, 91, 1772 - 1784.

(2) Jorio, A.; Dresselhaus, G.; Dresselhaus, M. Carbon Nanotubes: Advanced Topics in the Synthesis, Structure, Properties and Applications; Springer, 2008.

(3) Bindl, D. J.; Wu, M.-Y.; Prehn, F. C.; Arnold, M. S. Nano Letters 2011, 11, 455-460.

(4) Lefebvre, J.; Austing, D. G.; Bond, J.; Finnie, P. Nano Lett. 2006, 6, 1603-1608. 
(5) Cognet, L.; Tsyboulski, D. A.; Rocha, J.-D. R.; Doyle, C. D.; Tour, J. M.; Weisman, R. B. Science 2007, 316, 1465-1468.

(6) Georgi, C.; Böhmler, M.; Qian, H.; Novotny, L.; Hartschuh, A. Phys. Status Solidi (b) 2009, 246, 2683-2688.

(7) Siitonen, A. J.; Tsyboulski, D. A.; Bachilo, S. M.; Weisman, R. B. Nano Lett. 2010, 10, 1595-1599.

(8) Hertel, T.; Himmelein, S.; Ackermann, T.; Stich, D.; Crochet, J. ACS Nano 2010, 4, 71617168.

(9) Moritsubo, S.; Murai, T.; Shimada, T.; Murakami, Y.; Chiashi, S.; Maruyama, S.; Kato, Y. K. Phys. Rev. Lett. 2010, 104, 247402.

(10) Crochet, J. J.; Duque, J. G.; Werner, J. H.; Doorn, S. K. Nature Nanotech. 2012, 7, 126-132.

(11) Gokus, T.; Cognet, L.; Duque, J. G.; Pasquali, M.; Hartschuh, A.; Lounis, B. J. Chem. Phys. C 2010, 114, 14025-14028.

(12) Duque, J. G.; Pasquali, M.; Cognet, L.; Lounis, B. ACS Nano 2009, 3, 2153-2156.

(13) Cambré, S.; Santos, S. M.; Wenseleers, W.; Nugraha, A. R. T.; Saito, R.; Cognet, L.; Lounis, B. ACS Nano 2012, 6, 2649-2655.

(14) Berciaud, S.; Cognet, L.; Lounis, B. Phys. Rev. Lett. 2008, 101, 077402.

(15) Scott, D. W. Biometrika 1979, 66, 605-610.

(16) Xie, J.; Inaba, T.; Sugiyama, R.; Homma, Y. Phys. Rev. B 2012, 85, 085434.

(17) Xiao, Y.-F.; Nhan, T. Q.; Wilson, M. W. B.; Fraser, J. M. Phys. Rev. Lett. 2010, 104, 017401.

(18) Graham, M. W.; Ma, Y.-Z.; Green, A. A.; Hersam, M. C.; Fleming, G. R. J. Chem. Phys. 2011, 134, 034504. 
(19) Yoshikawa, K.; Matsunaga, R.; Matsuda, K.; Kanemitsu, Y. Appl. Phys. Lett. 2009, 94, 093109.

(20) Jiang, J.; Saito, R.; Samsonidze, G. G.; Jorio, A.; Chou, S. G.; Dresselhaus, G.; Dresselhaus, M. S. Phys. Rev. B 2007, 75, 035407.

(21) Shaver, J.; Crooker, S. A.; Fagan, J. A.; Hobbie, E. K.; Ubrig, N.; Portugall, O.; Perebeinos, V.; Avouris, P.; Kono, J. Phys. Rev. B 2008, 78, 081402.

(22) Perebeinos, V.; Tersoff, J.; Avouris, P. Nano Lett. 2005, 5, 2495-2499.

(23) Konik, R. M. Phys. Rev. Lett. 2011, 106, 136805.

(24) Kane, C. L.; Mele, E. J. Phys. Rev. Lett. 2004, 93, 197402.

(25) Jorio, A.; Fantini, C.; Pimenta, M. A.; Capaz, R. B.; Samsonidze, G. G.; Dresselhaus, G.; Dresselhaus, M. S.; Jiang, J.; Kobayashi, N.; Grüneis, A.; Saito, R. Phys. Rev. B 2005, 71, 075401 .

(26) Matsunaga, R.; Miyauchi, Y.; Matsuda, K.; Kanemitsu, Y. Phys. Rev. B 2009, 80, 115436.

(27) Duque, J. G.; Hamilton, C. E.; Gupta, G.; Crooker, S. A.; Crochet, J. J.; Mohite, A.; Htoon, H.; Obrey, K. A. D.; Dattelbaum, A. M.; Doorn, S. K. ACS Nano 2011, 5, 66866694.

(28) Munn, R. W.; Siebrand, W. J. Chem. Phys. 1970, 52, 47-63.

(29) Piryatinski, A.; Asher, S. A.; Mukamel, S. J. Phys. Chem. A 2002, 106, 3524-3530.

(30) Agranovich, V. M.; Konobeev, Y. V. Phys. Status Solidi (b) 1968, 27, 435-442.

(31) Capaz, R. B.; Spataru, C. D.; Ismail-Beigi, S.; Louie, S. G. Phys. Rev. B 2006, 74, 121401.

(32) Lüer, L.; Hoseinkhani, S.; Polli, D.; Crochet, J.; Hertel, T.; Lanzani, G. Nat. Phys. 2009, 5, 54-58. 